\title{
Probabilistic approaches and reliability design of power modules
}

\author{
A. Micol ${ }^{1, a}$, C. Martin ${ }^{1}$, O. Dalverny ${ }^{1}$, M. Mermet-Guyennet ${ }^{2}$ and M. Karama ${ }^{1}$ \\ 1 LGP-ENIT, 47 av. d'Azereix, BP 1629, 65016 Tarbes Cedex, France \\ 2 Power Electronics Associated Research Laboratory (PEARL), rue du Docteur Guiner, 65600 Semeac, France
}

Received 21 December 2007, accepted 25 March 2008

\begin{abstract}
The weak point for the standard power IGBT modules in terms of reliability is thermal fatigue in solder joints due to the thermal stress induced by constitutive materials with different coefficients of thermal expansion (CTE). So far, many researches are aimed at defining accurate finite element simulation with constitutive equations of materials behaviour and fatigue failure relation connecting the inelastic strain and the number of cycles before failure. Even if these relations can be clearly identified, we can see that the validation of the finite element model is difficult due to the scatter of input data. In fact, the fatigue life of solder joints strongly depends on geometric shape, solders behaviour (due to the process) and applied load. The aim of this paper is to estimate the probability of failure of power module with the structural reliability methods. Thus the geometric, materials and loading variables are considered as random variables and the failure mode is modelled with the called limit state function. The two methods, response surface method and neural network method, are used here to evaluate the reliability of the lead-free solder. The sensitivities of the mean and the standard deviation for each random variable have been evaluated.
\end{abstract}

Key words: Reliability; FORM; response Surface; IGBT; Sn/Ag solder.

\section{Introduction}

Finite element modelling is more and more used to achieve the reliability of electronics packages. The power of the computer capacity increasing, it is now possible to integrate more accurate definitions in finite element model such as materials characterization, refined mesh and effects on the surrounding to evaluate this reliability [1]. This can also be said of power semiconductor modules where their reliability becomes a main focus on current research. Moreover, the use of lead-free solder, with an accurate behaviour which still remains to investigate, becomes a crucial matter in environmental concerns. To improve the accuracy of modelization, the natural scatter and uncertainties existing in the real power module such as geometric dimensions or material properties, can be integrated in fatigue analysis. According to a model of fatigue, the sensitivity analysis can determine the relevant parameters that influence the failure $[2-4]$. To refine this sensitivity analysis, we have to integrate the data with a complete probabilistic definition and no longer as values defined in a range.

The reliability of this module being conditioned by the fatigue of the solder joints, the main factors influencing the latter are the materials properties and the shape of the solder $[5,6]$. One of the weak points for these modules

\footnotetext{
${ }^{a}$ Corresponding author: alexandre.micol@enit.fr
}

in terms of reliability was the wire-bonding connection, which is replaced here by a solution coming from the flip chip technology.

This paper presents the coupling between probabilistic and mechanical models to achieve fatigue life prediction for solders. First, we define and set up the probabilistic reliability methods and the mechanical-reliability coupling. Then, we present the mechanical problem by characterizing the mechanical and thermal behaviour of these connections with the constitutive law of the solder alloy. This behaviour is integrated as power law or hyperbolic sine law to calculate the stress-strain solution. The predictive fatigue life is estimated with the Coffin-Manson relation which uses equivalent inelastic strain in solder during one cycle. We analyse complete thermal and mechanical problems to achieve this reliability study, defining, on the one hand random variables as input of finite element model, and on the other hand a characteristic failure function.

\section{Probabilistic design method}

The probabilistic structural approach consists in determining, with a mathematical model, the probability of failure of a given system [7]. Indeed, when we build a traditional finite element model, all the model's input data are considered as fixed values and don't take into account 


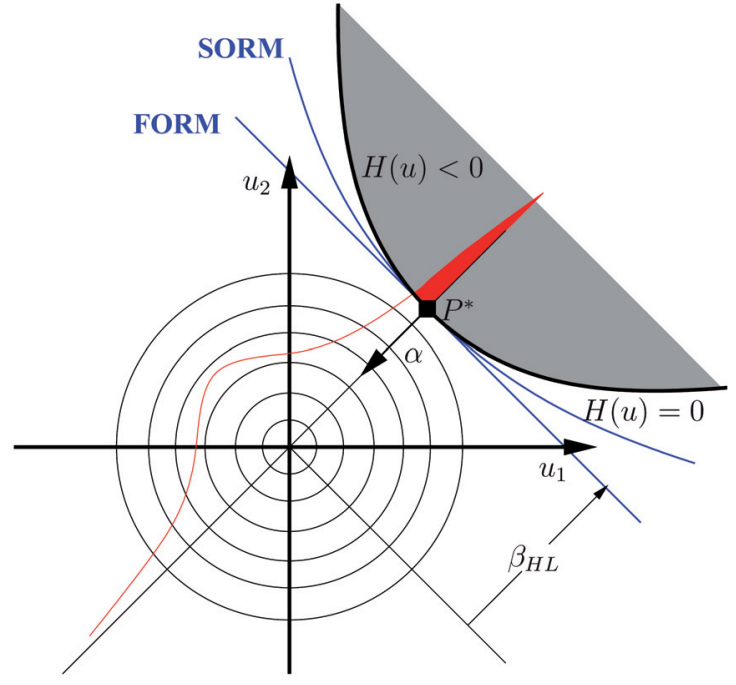

Fig. 1. Approximation methods FORM/SORM in standard space.

the natural scatter of the parameters. The solution to consider the natural variability and uncertainties of the input parameters on the model is to treat the input data as random variables defined by a law and its associated parameters. All relevant uncertainties influencing the probability of failure are then introduced in the vector $\boldsymbol{X}$ of basic random variables. In addition, the failure of the system is modelled by a functional relation $G(\boldsymbol{X})$, called limit state function, and defined to take a null or negative value in the failure domain:

$$
G(\boldsymbol{X}) \leq 0, \boldsymbol{X} \in \text { failure domain. }
$$

The components $x_{k}, 1 \leq k \leq n$ of the vector $\boldsymbol{X}$ are one realization of basic random variables. It's thus possible to define the probability of failure of the system as:

$$
P_{f}=\int_{G(X) \leq 0} f_{X}(x) d x_{1} \ldots d x_{n}
$$

where $f_{X}(x)$ is the $n$-dimensional probability density of basic random variable vector $\boldsymbol{X}$. The problem to solve this integral comes from the limit state function which is not explicit because its evaluation is the result of finite element call. Approximation method can be established to compute the multi-dimensional integral of equation (2) by substituting the limit state function for a linear or second order hyper plane called respectively First Order and Second Order Reliability Method (FORM and SORM) (Fig. 1).

The calculation scheme of approximation method is as follow and the code to achieve this task was inspired by Ferum [8]:

- to obtain a probability of failure independent from the different ways of writing the same limit state function, Hasofer-Lind [9] suggest to map $x_{i} \rightarrow u_{i}$ the basic random variables from physical space to independent standardized Gaussian space (Gaussian variable with null mean and unity standard deviation) with the transformation $T$. The limit state function is also mapped in standardized space: $G(X) \rightarrow H(U)$;

- the most probable failure point $P^{*}$, i.e. the nearest point to the origin belonging to the limit state in standardized space, is computed by the improved HasoferLind Rackwitz Fiessler (iHLRF) optimization algorithm [10,11];

- an approximation of limit state function is built with a hyper plane or a quadratic surface to the design point $P^{*}$ to compute the probability of failure with the relation:

$$
P_{f}=\Phi(-\beta)
$$

where $\beta$ is called the reliability index and $\Phi$ is the standard cumulative distribution function.

The problem of the design point algorithm in nonlinear finite element lies in the calculation of gradients. If the finite element code is not able to give the associated input derivatives, the latter have to be evaluated with the finite difference method. Indeed, the resolution of non-linear mechanical problems with finite element solver is achieved by integrating the non-linear materials laws. The use of an integration scheme involves an integration tolerance which defines the moment when the algorithm stops its iterations. For an accurate calculation of a stress increment, we define the error in the creep strain increment with:

$$
\Delta \varepsilon_{e r r}^{c r}=\left(\left.\dot{\bar{\varepsilon}}^{c r}\right|_{t+\Delta t}-\left.\dot{\bar{\varepsilon}}^{c r}\right|_{t}\right) \Delta t .
$$

This integration scheme can lead to incorrect values of gradient if the step of finite difference approximation is not taken with judicious value [12]. The solution then consists in finding an explicit function or method which can approximate the finite element output: the response surface method and neural network. A numeric design of experiments (DOE) is made to construct this response surface or to train the neural network. The neural network, which has the advantage not to take a specific shape of the output function, can however have a long training. In our study, we define both methods and compare these in the following application.

\subsection{Methodology}

There are two ways to construct the DOE, each of these comes with its own advantages and disadvantages. A full factorial design can be constructed in physic space or in standard space. Constructing the numerical design of experiments in physic space ensures that points are physically realistic and don't have an impossible mechanical situation. However, the points of DOE aren't always equally spaced in standard space (an accurate solution with iHLRF algorithm isn't ensured in the whole domain). On the other hand, the DOE constructed in normal space ensures a good equalised fitting in the entire normal space (a good conditioning which certifies the accurate solution) but it is possible that some points in 
normal space diverge with the Hasofer-Lind transformation. For our study, we'll construct the DOE in standard space.

\subsubsection{Building the response surface}

The response surface is constructed with random sampling of basic random variables following the uniform distribution defined in $[-3,3]$ in standard space. These points are then mapped in physical space and sent to the finite element software.

The response surface method is very well adapted to parallel computing: the different output calculation of the DOE with several input parameters can easily be parallelised on cluster. Each node of the cluster has a finite element calculation to solve and to return the output to the master. As opposed to traditional FORM method, where one node computes the output solution and the other nodes compute the gradient, with this method, all nodes are used to compute the response surface. The output is stored in a library to build the response surface function.

Finally, the surface method consists in approximating the limit state function $H$ with:

$$
\begin{aligned}
H_{r} & =H_{r}\left(u_{k}\right)=\eta\left(u_{k}, p\right)+\varepsilon_{r} ; \\
\mathrm{E}\left(\varepsilon_{r}\right) & =0 ; k=1, . ., n ; r=1, . ., N
\end{aligned}
$$

where $H_{r}$ is the $r$ th evaluation of the exact limit state function at the $u_{k}$ point, $\eta\left(u_{k}, p\right)$ the response surface with the $p$ parameters at the $u_{k}$ point and $\varepsilon_{r}$ the error between the limit state function and the response surface.

For this study, we're going to construct different surface functions such as linear (polynomial) and non-linear regression models.

In the case of linear regression problems, the response surface for the $r$ th sample can be written as follows:

$$
\eta^{(r)}\left(u_{k}\right)=p_{0}+\int_{j=1}^{m} p_{j} \Psi_{j}\left(u_{k}\right)
$$

which, when using pure matrix notation for all sample, remains:

$$
\eta=\Gamma \theta
$$

where $\eta$ contains the different responses $\eta^{(r)}\left(u_{k}\right), \Gamma$ represents the data matrix of DOE and $\theta$ the vector containing the parameters $p$.

The fitting method consists in finding $\bar{\theta}$, the vector of unknown coefficients $p$, by minimizing the sum of squares:

$$
\bar{\theta}=\min _{p}\|H-\eta\|^{2}
$$

and is solved by:

$$
\bar{\theta}=\left(\Gamma^{T} \Gamma\right)^{-1} \Gamma^{T} \eta .
$$

In the case of nonlinear regression problems, the data matrix cannot be built and the Levenberg-Marquardt algorithm is used to solve equation (8).
The relevance of the prediction model can be evaluated with an analysis of variance. The multiple regression correlation coefficient $\bar{R}^{2}$ is a measure of the proportion of variability explained by, or due to the regression (linear relationship) in a sample of paired data:

$$
\bar{R}^{2}=1-\frac{\sum_{i}\left(H_{r}\left(u_{k}\right)-\eta^{(r)}\right)^{2} /(N-m-1)}{\sum_{i}\left(E\left[H_{r}\left(u_{k}\right)\right]-\eta^{(r)}\right)^{2} /(N-1)} .
$$

This coefficient can lead error when the shape of response surface is unknow. We introduce for that the predictive quality of the regression can be evaluated by the $Q^{2}$ index:

$$
Q^{2}=1-\frac{\sum_{r=1}^{N} \epsilon_{r}^{2}}{\sum_{r}\left(E\left[H_{r}\left(u_{k}\right)\right]-\eta^{(r)}\right)^{2}}
$$

where:

$$
\epsilon_{r}=\frac{H_{r}\left(u_{k}\right)-\eta^{(r)}}{1-\mathbf{H}_{i i}}
$$

and where $\mathbf{H}$ is the hat matrix defined by:

$$
\mathbf{H}=\Gamma\left(\Gamma^{T} \Gamma\right)^{-1} \Gamma^{T} .
$$

In the the case of non-linear regression problems, a linearization of the matrix of experiments is made around the supposed vector of parameter $\theta$ :

$$
\Gamma=\left(\frac{\partial \eta(u, p)}{\partial p_{1}}, \ldots, \frac{\partial \eta(u, p)}{\partial p_{n}}\right) .
$$

This can correctly made with a prior knowledge of parameters $p$. The different iterations for finding the design point use then the previous parameters of the reponse surface for assess the current matrix of experiments. The fisrt iteration use as for it the defined the start $\theta$ vector.

We present here an optimization algorithm for establishing the design plan with continue and delimited input. The method presented here consists to solve the optimization problem:

$$
\begin{aligned}
\mathcal{L}(u, \lambda)= & -\min _{u^{(i)}, u^{(j)} \forall i, j}\left(d\left(u^{(i)}, u^{(j)}\right)\right) \\
& +\lambda_{0}\left[\mathbf{H}_{\max }-\operatorname{Tr}\left(\mathbf{H}-\mathbf{I} \frac{N}{m+1}\right)\right] \\
& +\sum_{i} \lambda_{i}\left[u_{\max }-\left\|u^{(i)}\right\|\right]
\end{aligned}
$$

where:

- $\mathcal{L}(u, \lambda)$ represents the lagrangian with the $\lambda$ multiplicators;

- $-\min _{u^{(i)}, u^{(j)}}\left(d\left(u^{(i)}, u^{(j)}\right)\right)$ represents the function which maximize the minimal distance between two points;

$-\mathbf{H}_{\max }-\operatorname{Tr}\left(\mathbf{H}-\mathbf{I} \frac{N}{m+1}\right)$ represents the constrain to not obtain influent point;

$-u_{\max }-\left\|u^{(i)}\right\|$ represents the constrain on the norm of the vector $u^{(i)}$ to prevent them from diverging beyond the cercle with a radius $u_{\max }$. 


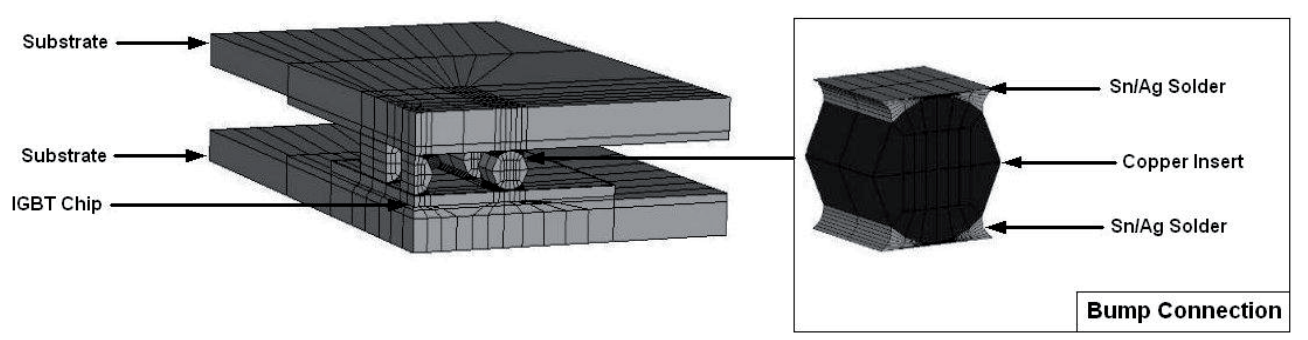

Fig. 2. The 3-D finite element model.

The algorithm starts to fit the points with a number of finite element call equal to the number of fitted parameters. These points are optimized in the domain by solving equation (11) with the COBYLA algorithm. At this point, the degree of freedom of the model $(N-m-1)$ is equal to 0 . The approximation cannot be relevant, the algorithm then optimizes and computes other points in the domain until it obtains $Q^{2}$ index higher than 0.9 to ensure a relevant model.

\subsubsection{FORM analysis with explicit function}

After having approximated the limit state function with response surface or neural network, the FORM method described above is used. To find an accuracy design point, the response surface method or neural network coupling with the design point optimisation is repeated with, at each step, a reduction of the DOE space. In fact, after finding the design point with the explicit function, a real finite element solution is computed at this point and, if this point doesn't respond to the convergence condition, another DOE is made around this point. The previous points stored in the library are used if their coordinates belong to the new design of experiments space.

\subsubsection{Sensitivities}

The main results available with approximation methods is then the probability of failure of the system (Eq. (3)) but other interesting results are the sensitivities of this probability regarding the different basic random variables [13]. In FORM formulation, the limit state function can be written $H(u)=a u+\beta$ under normalized form. We can then define the sensitivity of the reliability index with respect to the standardized random variables as follows:

$$
\alpha=\nabla_{u} \beta=\left[\frac{\partial \beta}{\partial u_{1}}, \frac{\partial \beta}{\partial u_{2}}, \ldots, \frac{\partial \beta}{\partial u_{2}}\right]=\left.\frac{\nabla_{u} H(u)}{\left\|\nabla_{u} H(u)\right\|}\right|_{u^{*}} .
$$

We can study the sensitivity $\alpha_{p_{i_{\gamma}}}$ of the reliability in$\operatorname{dex} \beta$, and then the probability of failure, with respect to the distribution parameters, such as mean or standard deviation, of the $\gamma$ th random variables to identify those which must have stricter quality control. The sensitivity $\alpha_{p_{i_{\gamma}}}$ is calculated with:

$$
\nabla_{p} \beta=-J_{u^{*}, p} \alpha
$$

where $J_{u^{*}, p}(i, j)=\frac{\partial T_{i}\left(x^{*}, p\right)}{\partial p_{j}}$ is the Jacobian matrix between the Hasofer-Lind transformation and the parameter $p$.

This sensitivity cannot be used directly for comparison, so the elasticity is defined by the normalisation of this sensitivity:

$$
e_{p_{i}}=\frac{p_{i}}{\beta} \alpha_{i_{\gamma}} \text { with } \alpha_{i_{\gamma}}=\left.\frac{\partial \beta}{\partial p_{i_{\gamma}}}\right|_{u^{*}} .
$$

\section{FE modelling}

\subsection{Geometry model}

The device studied is a new water cooled IGBT power module. This module includes four IGBT chips and two diodes brazed on a substrate with a two-side cooling. The chip is cooled through a water blade on the top substrate and by a heat sink on the lower substrate. The power IGBT module FE model simulates one IGBT soldered on Aln substrate and attached with the top substrate with bump connection. A lead solder is used to solder the chip with the substrate and the eutectic $\mathrm{Sn} / \mathrm{Ag}$ alloy is used to joint the two substrates via copper bumps cylinder-shape (1.45 mm diameter and $2 \mathrm{~mm}$ long) and two solders. Figure 2 shows a quarter of the assembly with bump technology. The bump connection is soldered to the IGBT with gold layer, and to the top substrate with $250 \mathrm{~mm}$ nickel metallization. Only a quarter of the entire multilayer switch is used because of the symmetric boundary condition and mesh optimization.

We define here the variability of the geometric dimensions. Only the shape of the solder is assumed to evolve during the process. This study focuses on the geometric dimension of the bump connection. The cross section of the bump connection in Figure 3 highlights the different geometric dimensions. Table 1 reports the characteristic geometric dimensions of the bump connection made on 20 specimens according to the dimension reported in Figure 3. Their mean value and standard deviation, calculated according to the geometric dimensions, follows a log-normal distribution. Only the thickness $e$ between chip and copper insert seems to have a relevant influence on the failure. This dimension is then included in probabilistic analysis. 


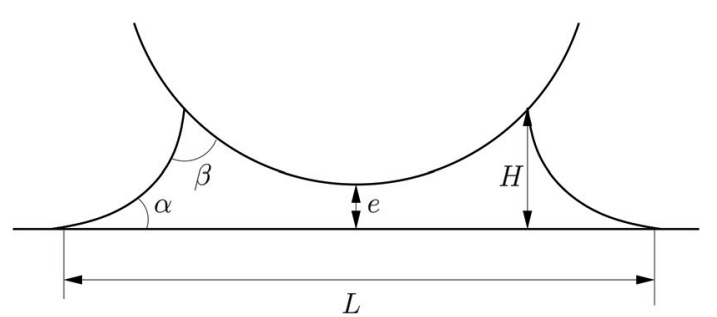

Fig. 3. Dimension characterising the solder bump.

Table 1. Geometric dimension results of cross section of bump connection according to Figure 3.

\begin{tabular}{lll}
\hline Geometric dimension & mean & Standard deviation \\
\hline solder thickness $(e)$ & $12 \mathrm{~mm}$ & $6 \mathrm{~mm}$ \\
solder height $(h)$ & $175 \mathrm{~mm}$ & $16 \mathrm{~mm}$ \\
$\alpha$ & 32 & 7 \\
$\beta$ & 48 & 8 \\
solder pad size $(L)$ & $1087 \mathrm{~mm}$ & $39 \mathrm{~mm}$ \\
\hline
\end{tabular}

\subsection{Materials characterisation}

Lead free alloy is used as bump solder in the present module. The elastic modulus is dependent on the temperature, and can be modelled with the following equation:

$$
E=E_{0}-E_{1} T\left({ }^{\circ} \mathrm{C}\right)
$$

with $E$ the temperature dependent Young modulus in $\mathrm{MPa}$, the Young modulus at $0{ }^{\circ} \mathrm{C}$, the decrease rate of the Young modulus according to the temperature and $T$ the temperature in degree Celsius. For our solder, several nano-indentations are made in the solder to evaluate, which is taken as materials random variables in the probabilistic study.

The material non-linearity is modelled with the constitutive law characterizing the steady state creep rate. The power law can describe the mechanical behaviour of alloy in low or medium stress:

$$
\dot{\varepsilon}_{c r}^{\cdot}=A(B \sigma)^{n} \exp \left(\frac{-Q}{k T}\right)
$$

with $\varepsilon_{c r}$ the equivalent creep strain rate, $\sigma$ the applied stress and $T$ the temperature. Wiese identifies the law's different parameters for the eutectic $\mathrm{Sn} / \mathrm{Ag}$ solder alloy in flip-chip configuration [14]. When high stress is reached the power law breaks down and can't follow the creep strain rate to increase more quickly with the applied stress. A second law was developed to find a closed form of creep behaviour. The sine hyperbolic law can describe the steady state creep for the low and high stress level:

$$
\dot{\varepsilon}_{c r}=A(\sinh (B \sigma))^{n} \exp \left(\frac{-Q}{R T}\right) .
$$

This sinh law, identified for Sn/Ag3.5 and Sn95.5/Ag3.8/Cu0.7 by Banerji and Darveaux [15], is presented with the different parameters. For our application and stress level, the best way should be to use the sine hyperbolic law but Wiese demonstrates the strong dependence of the law's parameters on the solder configuration during identification. To compute a best approach to the creep strain for flip-chip configuration, the identified flip-chip power law would be more suitable. The multiplier coefficient $A$ and the power coefficient $n$ are considered as random variables and are assumed to have a variation coefficient of $10 \%$.

We consider the solder to have an isotropic linear strain hardening. The plasticity's parameters are incorporated in the study but are not considered as random variables.

\subsection{Thermal load}

The faces of the heat sink wings are subjected to thermal convection with a convective exchange coefficient calculated at the beginning, starting from a fluid software. The power IGBT module is subjected to a power cycling history. The active power cycling consists in computing a primary heat transfer analysis and the thermal temperature map output is sent to the mechanical analysis. The power injected is distributed in the volume of the IGBT. For this power cycling, temperature time history loading is only imposed to the element corresponding to the IGBT. Figure 4 shows the output temperature of the heat transfer analysis sent to the mechanical analysis to compute the difference of the output inelastic strain between the end and the beginning of the limit cycle.

\subsection{Life prediction}

The models proposed to evaluate predictive fatigue life of the solder joints can be divided in five major categories based on stress, plastic and/or creep strain, energy and damage accumulation [16]. For our study, we take the inelastic strain based model which considers plastic and creep phenomenon due to CTE mismatch. The following equation shows the Coffin-Manson $[17,18]$ fatigue life model:

$$
N_{f}=C \Delta \varepsilon_{i n}^{n}
$$

where is the accumulated equivalent inelastic strain during one cycle. For Sn/Ag3.5, $C=23.78$ and $n=$ -1.08 [19] and for Sn95.5/Ag3.8/Cu0.7, $C=0.38$ and $n=-1.96[20]$. The evaluation of the number of cycles before failure is investigated considering the whole volume of the solder. To calculate the accumulated equivalent inelastic strain, we use the following averaged equation with the volume weighted average (VWA) method:

$$
\Delta \varepsilon_{i n}=\frac{\sum_{e} \Delta \varepsilon_{i n_{e}} V_{e}}{V_{t o t}}
$$

where $V_{t o t}$ is the total volume of the elements' set, $V_{e}$ is the volume of one element and $\Delta \varepsilon_{i n_{e}}$ the associated inelastic strain. This method ensures that the solution is less mesh dependent compared to using the maximum value of inelastic strain in the whole solder. 

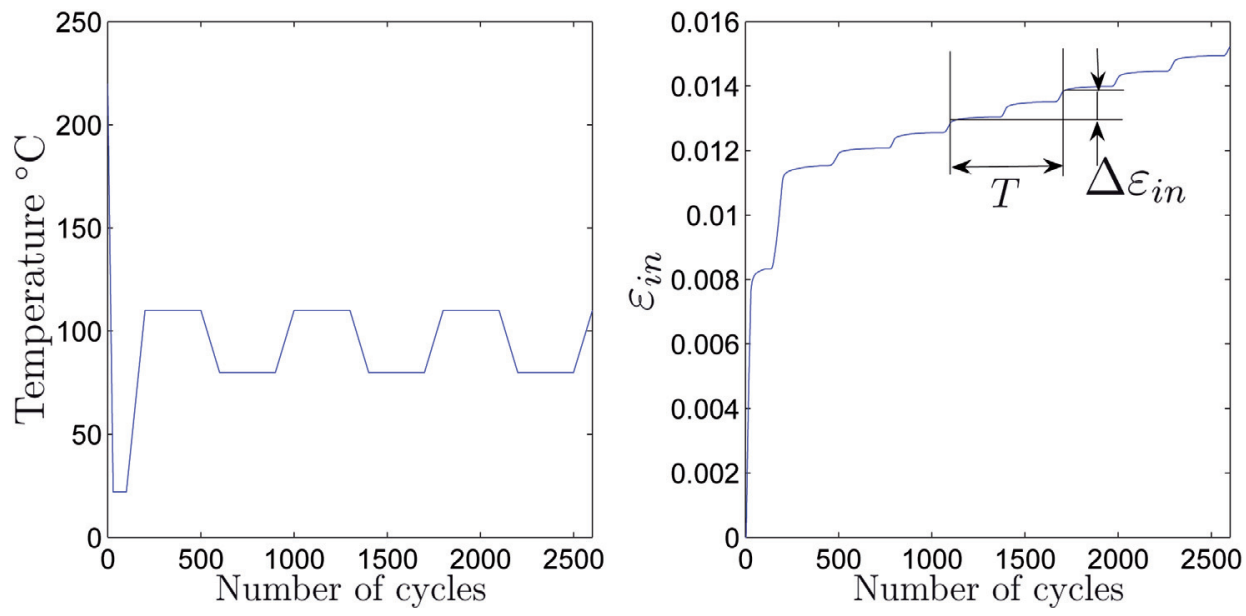

Fig. 4. Result temperature in chip and associated inelastic strain.

\subsubsection{Random variable and limit state function}

The limit state function, relating the thermo-mechanical fatigue of the solder, is written considering that the system falls into the failure field if the number of cycle doesn't reach a target value. The mechanical output in terms of inelastic strain is transformed by using the Coffin-Manson relation:

$$
G(X)=C \Delta \varepsilon_{\text {in }}^{n}-N_{f_{\text {target }}}
$$

where $C \Delta \varepsilon_{\text {in }}^{n}$ and $N_{f_{\text {target }}}$ are respectively the number of cycles before failure computed by the finite element code and the objective number of cycles that the system must reach.

\subsection{Results}

The sensitivities study shows the evolution of the probability of failure and the importance of thermal solicitation. The main parameter influencing the failure remains the flux density imposed in the IGBT chip. The multiplier coefficients $A$ of the constitutive law slightly act on the results of inelastic strain. The effort for an accurate identification must be made on the power coefficient $n$ because of the influence of its mean value: the recommendation of the previous authors about a micro structure (varying with the temperature cooling and then the shape of solder due to this capacity of cooling) must be taken into account. Moreover, the sensitivity of this standard deviation indicates that a variability in the process or a variability on the quality of solder have a great impact in terms of reliability. The Sn/Ag CTE mean value and its standard deviation, which intervene in the failure, don't show a great dependence on the probability of failure.

The main design variability of this bump connection technology is identified on the thickness $e$ because this is the only one of the geometric dimensions, identified with the experiments, which shows great correlation with the failure. It leads to a better definition of the shape and

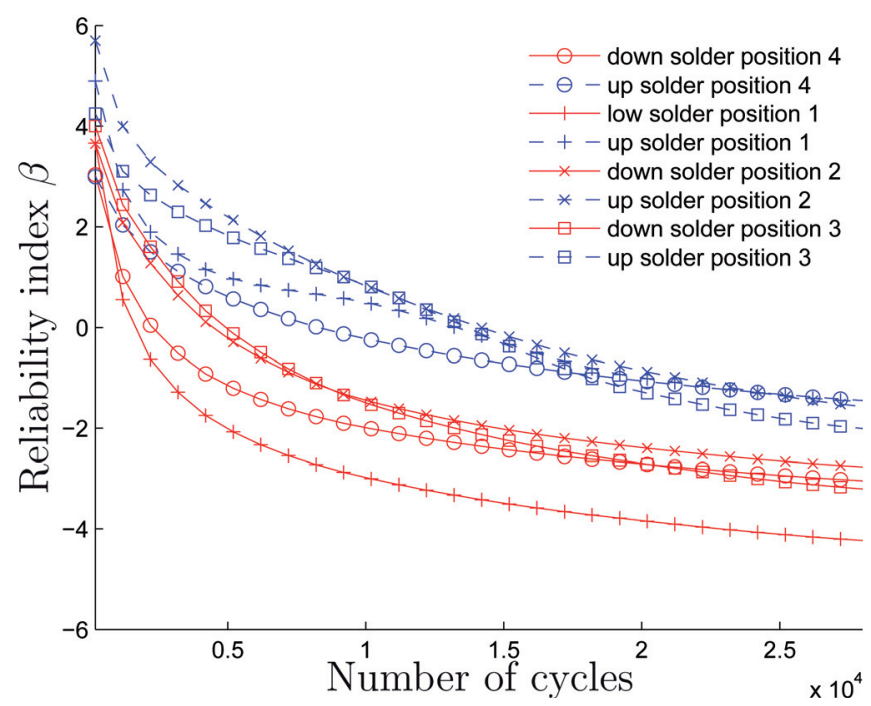

Fig. 5. Evolution of the reliability index $\beta$ for the target number of cycles.

especially the problem of the location of the initial crack. Figure 5 shows the evolution of the $\beta$ reliability index and Figure 6, the probability of failure of the module for different numbers of cycle and for the different solders of the module. It must keep in mind that the initial crack begins under the bump but in terms of electric functionality, the failure is not reached.

An investigation is now made to compare first mechanic formulations of the solder behaviour and then, different solders. The reliability is evaluated with the sinus hyperbolic and power behaviour formulation for $\mathrm{Sn} / \mathrm{Ag} 3.5$ solder and with a sinus hyperbolic for Sn95.5/Ag3.8/Cu0.7. The number of cycles to reach to be in safe domain is taken here at $N_{f_{\text {target }}}=2000$ cycles. The response surface is used here. For the same limit state function using the same coefficient of the Coffin-Mason relation, a difference is found according to the creep constitutive equation (Eqs. (16) and (17)). The main difference, highlighted by Figures 7 and 8, between the two 


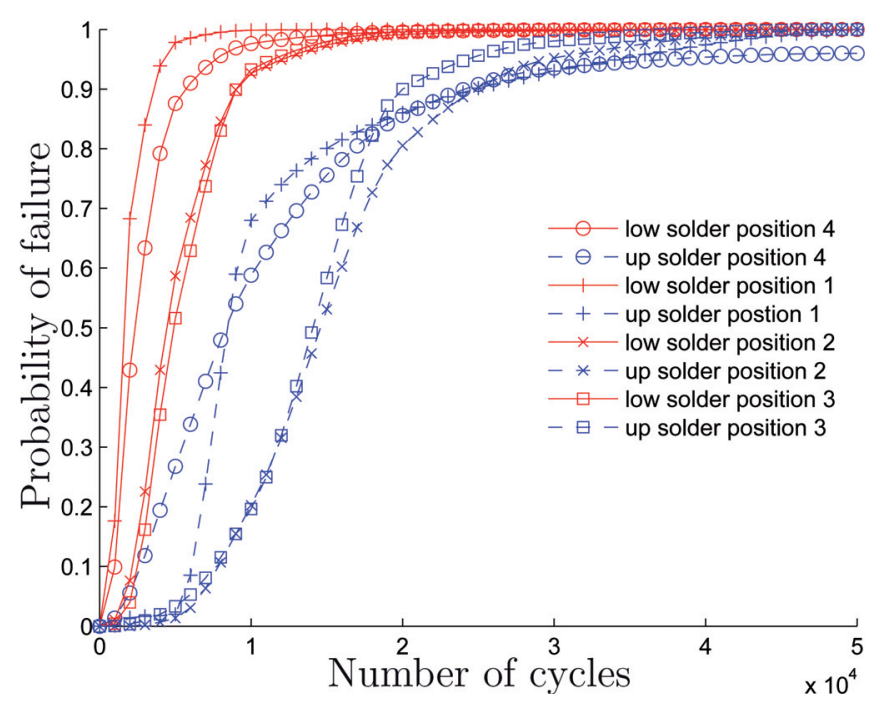

Fig. 6. Evolution of the probability of failure for the target number of cycles.

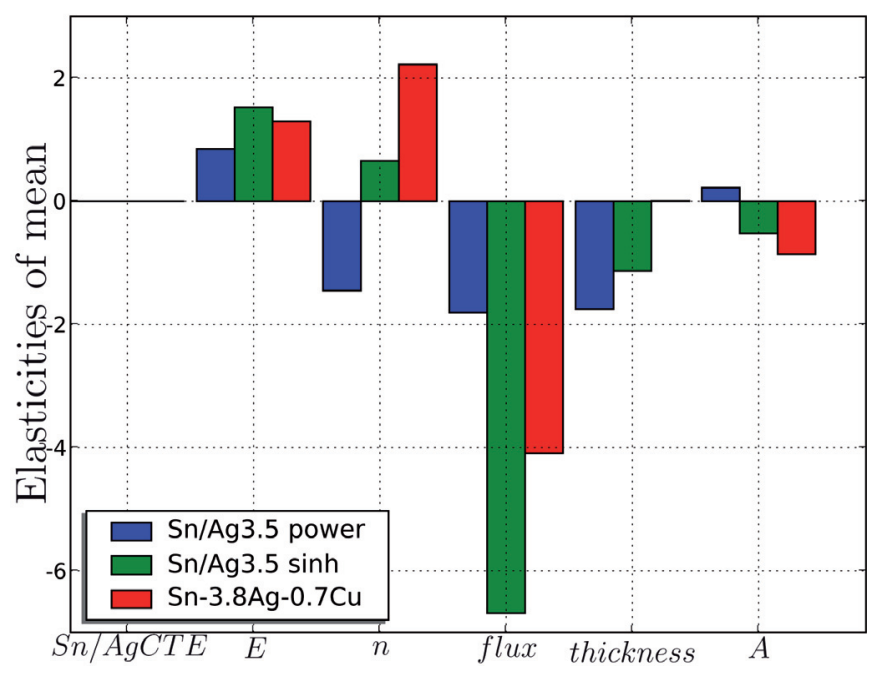

Fig. 7. Elasticities of the random variable's mean for different solder alloys.

formulations lies in the fact that the two laws are not identified on the same specimen. It is confirmed that the identification procedure of the law's parameters plays a major role. The Sn95.5/Ag3.8/Cu0.7 denotes a relatively better reliability in comparison to the $\mathrm{Sn} / \mathrm{Ag} 3.5$ solder if we consider the same constitutive law that corresponds to Schubert's result. It is reminded that the Coffin-Masson parameters play a role here too.

\section{Conclusion}

We attempt in this article to estimate the reliability of power modules connections with structural reliability methods. The two approximation methods, response surface and neural network, are set up and used to define the probability of failure and the sensitivities of this latter ac-

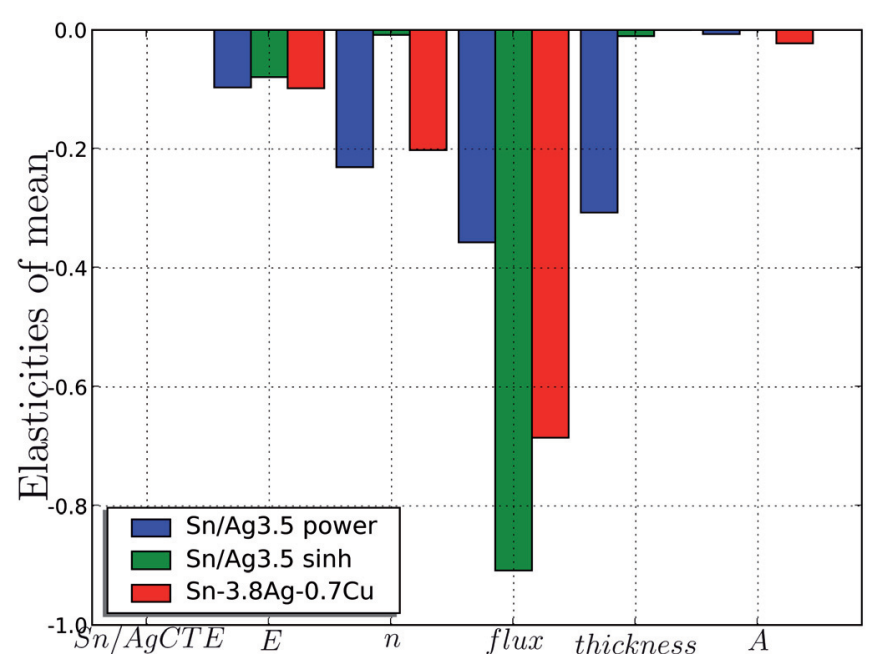

Fig. 8. Elasticities of the random variable's standard deviation for different solder alloys.

cording to the random variables. The response surface can model the behaviour of the inelastic strain if we can define the shape of the function. The advantage of neural network to not know the shape of the model is to solve this issue but it can be a problem because of the number of finite element call. The reliability analysis was made by taking as random variables the characteristics of the different materials. It shows the importance of the power law coefficient $n$ but a variation of the loading action remains the major parameter changing the reliability. The future developments will have to include more sophisticated integration of load behaviour such as rainflow counting. We must keep in mind that this method evaluates the failure according to one failure mode: the fatigue of solders. The results of this reliability investigation remain sensitive to the constitutive law, its identification and to the fatigue failure relation.

\section{References}

1. P. Towashiraporn, G. Subbarayan, B. McIlvanie, B.C. Hunter, D. Love, B. Sullivan, in Thermal and Thermomechanical Phenomena in Electronic Systems (ITHERM, 2002), pp. 854-861

2. B. Vandevelde et al. Journal of Electronic Packaging 125, $498(2003)$

3. D.G. Yang, J.S. Liang, Q.Y. Li, L.J. Ernst, G.Q. Zhang. Microelectronics Reliability 44, 1947 (2004)

4. B. Wunderle, W. Nuchter, A. Schubert, B. Michel, H. Reichl. Microelectronics Reliability 44, 1933 (2004)

5. L. Xu, T. Reinikainen, W. Ren, B.P. Wang, Z. Han, D. Agonafer. Microelectronics Reliability 44, 1977 (2004)

6. L. Xingsheng, L. Guo-Quan, in Components and Packaging Technologies, IEEE Transactions (see also Components, Packaging and Manufacturing Technology)

7. R. Rackwitz. Structural Safety 23, 365 (2001)

8. T. Haukass, Finite element reliability using matlab, http://www.ce.berkeley.edu/haukaas/FERUM/ ferum.html 
9. N.C. Lind, A.M. Hasofer. J. Eng. Mech. 100, 111 (1974)

10. B. Fiessler, R. Rackwitz. Comput. Struct. 9, 489 (1978)

11. A. Der Kuireghian, P.L. Liu. Struct. Safety 9, 489 (1991)

12. A. Micol, M. Karama, O. Dalverny, C. Martin, M. Mermet-Guyennet, in Reliability design of power modules using probabilistic approaches, edited by B.H.V. Topping, G. Montero, R. Montenegro, Proceedings of the Eighth International Conference on Computational Structures Technology (Stirlingshire, United Kingdom, Civil-Comp Press, 2006)

13. M. Lemaire, Fiabilité des structures (Hermes, Collection Génie civil, 2005)

14. E. Meusel, S. Wiese, F. Feustel. Sensors and Actuators 99, $188(2002)$
15. K. Banerji, R. Darveaux, Constitutive relations for tinbased solder joints, in IEEE Proceeding (1992), pp. 10131024

16. G.S. Selvaduray, W.W. Lee, L.T. Nguyen. Microelectronics Reliability 40, 231 (2000)

17. L.F. Coffin. J. Mater. 6, 388 (1971)

18. S.S. Manson, Thermal Stress and Low-cycle Fatigue (New York, Mc-Graw-Hill, 1966)

19. C. Kanchanomai. International Journal of Fatigue 24, 57 (2002)

20. A. Schubert et al., in Electronic Components and Technology Conference (Proceedings 52nd, 2002), pp. 1246-1255 